\title{
El role-play como ejemplo de aprendizaje duradero en población adulta
}

\author{
Ernesto José Peris Chanzáa ${ }^{a, b}$ \\ ${ }^{a}$ Experto docente para el SERVEF dentro del programa formativo "Operaciones auxiliares y de almacén en industrias \\ y laboratorios químicos (QUIE0308)". "Profesor en el Centro Psicopedagógico UNICS (Alcàsser, Valencia). \\ Contacto: eperisch@gmail.com
}

\begin{abstract}
In this paper the results achieved regarding long-term knowledge and concept remembrance collected by online interview one year after the finishing of the course "Auxiliary and warehouse operations in chemical industries and laboratories" are shown. The work is focused on role-play learning methodologies versus other so-called traditional strategies, with the aim at gaining evidences on those methods of information handling as more efficient and reliable for the learned concepts to be understood more deeply and interiorized for a longer time. In this sense, one year after completing the course, the students were interviewed with questions related to some of the matters performed under both types of confronted approaches. The results seem well aligned with the main thesis that lays at the foundation of role-play as a useful learning method: when students take a starring role in their own learning process, the concepts are absorbed and integrated in a more durable fashion (in contrast to the traditional passive learning). The data here displayed are more relevant when taken into account that most of the participants did not possess any previous ideas on the subjects dealt within this course.
\end{abstract}

Keywords: role-play, role dynamics, debates, adult learning, interactive methodologies through information exchange.

\section{Resumen}

En este trabajo se presentan los resultados obtenidos respecto a retención de conocimiento a largo plazo que fueron recopilados por el método de encuesta on-line un año después de la finalización del curso "Operaciones auxiliares y de almacén en industrias y laboratorios químicos". El trabajo está orientado a la experimentación con metodologías role-play frente a otras estrategias tradicionales de aprendizaje, con la intención de obtener evidencias sobre aquellos métodos de manejo de información como más eficientes y fiables a la hora de asimilar y comprender los conceptos de manera más profunda y duradera. En este sentido, los estudiantes fueron entrevistados un año después de terminar el curso con preguntas sobre conceptos trabajados con ambos tipos de métodos. Los resultados obtenidos apuntan en la línea de la tesis fundamental de la técnica role-play como método útil de aprendizaje: cuando los estudiantes asumen un papel protagonista en su propio proceso de aprendizaje, las ideas resultan absorbidas e integradas de manera más duradera (frente al aprendizaje pasivo tradicional). Los resultados aqui presentados poseen mayor relevancia, si cabe, al considerar que gran parte de los estudiantes no tenían un conocimiento previo en las materias impartidas.

Palabras clave: role-play, dinámica de rol, debate, aprendizaje en adultos, metodologías interactivas de intercambio de información. 


\section{Introducción}

La entrada en la era digital (dejando atrás la industrialización y la revolución tecnológica) está provocando que todos los aspectos de la vida del ser humano necesiten ser adaptados y reformulados según las nuevas normas y modos de interacción social, laboral y personal. En este sentido, también el proceso de enseñanza-aprendizaje está experimentado cambios en los últimos tiempos. El paradigma del docente como foco de conocimiento está dando paso (aunque, en nuestra opinión, no lo suficientemente deprisa) a una enseñanza centrada en el camino del estudiante por interiorizar de manera motivada y consciente tanto ideas como procedimientos (Reeves y col. 2002).

A este respecto está siendo de gran ayuda el compromiso de las instituciones europeas y de los gobiernos nacionales en la redacción del marco normativo que regule estos cambios y, principalmente, de la incorporación al ámbito educativo del concepto de Competencias Claves: "Son aquellas competencias que todas las personas precisan para su realización y desarrollo personal, así como para la ciudadanía activa, la inclusión social y el empleo". Se trata de un conjunto de siete habilidades que se han considerado esenciales para la formación de los ciudadanos del siglo XXI, a saber: comunicación lingüística; competencias matemática, científica y tecnológica; competencia digital; competencias sociales y cívicas; iniciativa y espíritu emprendedor; conciencia de expresiones culturales; y, por último, aprender a aprender. El trabajo de estas competencias debe enfocarse desde una óptica transversal y dinámica que permita un crecimiento personal armonizado para conseguir que los estudiantes adquieran, a lo largo de las distintas etapas académicas, las capacidades de saber hacer (conocimiento procedimental), saber decir (conocimiento declarativo) y saber social (conocimiento social) (ECD/65/2015, RD 1105/2014, D 51/2018).

Los contenidos y recursos ya no deben ofrecerse como una clase magistral sino de manera que fomenten el aprendizaje participativo y autónomo donde el estudiante tome el mando de su propia formación. Aquí es donde los docentes deben hacer un esfuerzo notable en el diseño de los materiales y en la forma de ofrecerlos procurando, además, construir un nexo entre las actividades propuestas, la evaluación de las mismas y los logros de aprendizaje conseguidos (Biggs y col., 2011) que se alimente del diálogo constante profesor-estudiante (Coffield, 2008).

Uno de los factores principales a tener en cuenta cuando se diseña el aprendizaje de un concepto es que el estudiante se sienta interesado por la propuesta (o, idealmente, que sea él mismo quien escoja el concepto que quiere trabajar). Debe querer resolver una pregunta que le intriga y le lleva a ponerse en marcha (Race, 2010). Se debe dar el salto del aprendizaje pasivo al aprendizaje activo y motivado. Aquí es donde entran en juego las metodologías role-play. Esta aproximación al proceso enseñanza-aprendizaje puede incorporar muchos de los aspectos requeridos a nivel normativo y considerados esenciales en la formación de los actuales estudiantes: se trata de un enfoque activo donde cada estudiante debe asumir un papel (rol) e interaccionar con sus otros semejantes. En resumen, se ponen en marcha los conocimientos fundamentales procedimental, declarativo y social para conseguir, de manera cooperativa, un objetivo común pero también individual. La limitación en la aplicación de esta metodología depende del nivel de imaginación de docentes y alumnos, pudiéndose aplicar a cualquier ámbito formativo, nivel académico o materia (McSherry y col., 2000). Desde literatura, historia, ciencias o lenguas, cualquier disciplina puede adaptar alguna de las herramientas role-play en el trabajo de sus conceptos. Valgan como ejemplo dinámicas de grupo, aprendizaje por proyectos (con roles asignados dentro de cada grupo), dramatización de situaciones, interacción directa con el entorno social, construcción material de los productos de aprendizaje (montaje de un vídeo, edición de un podcast, participación en una actividad municipal, etc.). 


\section{E. Peris Chanzá}

\section{Objetivos}

Los objetivos principales del estudio que se presenta fueron:

a. Implementar el uso de herramientas role-play en el trabajo de determinados conceptos dentro de un curso para personas adultas (debates tomando posiciones "forzadas", dramatización, dinámicas de rol).

b. Desarrollar determinados conceptos en sesiones presenciales de una manera más dinámica y entretenida.

c. Comprobar el grado de implicación y motivación de los participantes en actividades desarrolladas con metodología role-play frente a metodologías clásicas.

d. Analizar los resultados de retención de conceptos a largo plazo comparando el enfoque roleplay y tradicional.

\section{Desarrollo de la innovación}

\subsection{Grupo de estudio.}

El trabajo se llevó a cabo sobre un grupo de 12 estudiantes, aquellos que completaron el curso y superaron la evaluación con éxito. Se trataba de un conjunto de personas muy heterogéneo en cuanto a que poseían experiencias laborales previas muy diferentes unos de otros (desde operarios de almacén logístico, a comerciales, pasando por auxiliares de residencia, técnicos de laboratorio o técnicos agrícola) así como a distintos niveles académicos superados (el acceso al curso requería que se acreditase como mínimo una titulación de ESO o FPB; a partir de aquí, el grupo abarcaba desde personas con titulación ESO, otras con FP de grado medio y dos licenciaturas en áreas experimentales).

\subsection{Curso y sesiones.}

El curso estaba organizado por el Servicio Valenciano de Empleo (SERVEF, recientemente renombrado LABORA), dentro del Programa de Políticas Activas de Empleo dirigidas a la mejora de las posibilidades de reincorporación al mercado laboral de personas físicas en situación de desempleo, en colaboración con el Centro Integrado Público de Formación Profesional CIPFP Vicente Blasco Ibáñez de Valencia, que ofreció sus instalaciones (aulas y laboratorios) y recursos tanto materiales (reactivos, material de oficina y fotocopias) como personales (desde dirección del centro a jefatura de estudios y profesores del departamento de Química,) para la realización de las actividades.

El curso tuvo una duración de 310 horas, dedicándose $270 \mathrm{~h}$ al desarrollo de los contenidos teóricos detallados en el Real Decreto RD 719/2011 (20 de mayo) referidos al Certificado de Profesionalidad para el que capacita la superación de este curso "Operaciones auxiliares y de almacén en industrias y laboratorios químicos" (con código QUIE0308), agrupados en cuatro Módulos formativos que contemplaron las distintas Unidades de competencia que el alumnado debía necesariamente conocer y practicar para superar el curso. Los últimos treinta días se emplearon en la realización de prácticas en empresa así como en la evaluación final del curso mediante un examen teórico-práctico y en la emisión de las actas y certificados oportunos.

Cada sesión del curso tenía una duración de 4 horas que se distribuía en función de las necesidades del contenido en una parte teórica, una sesión práctica (cuando era necesario) y una sesión de dinámica grupal. 


\subsection{Actividades.}

Aquí presentamos algunas de las actividades que se desarrollaron durante el curso atendiendo a los ámbitos cognitivos y al tipo de pensamiento que el estudiante debe aplicar para la comprensión de conceptos y el avance en el temario (Bloom y col., 1956; Anderson y col., 2001).

\subsubsection{Enfoque clásico.}

Estas actividades se enmarcaron en los ámbitos cognitivos de "recordar y aprender" en los cuales el estudiantes debe aplicar un tipo de pensamiento de orden inferior (LOT, son las siglas en inglés para Low Order Thinking). Este tipo de enfoque se caracteriza por el uso de metodologías fundamentadas en acciones del tipo: recordar, etiquetar, identificar, memorizar, describir, localizar, recuperar, listar, resumir, anotar, asociar, generalizar, reordenar, distinguir, comparar, etc. (theflippedclassroom.es, 2015) datos y respuestas sin necesidad de profundizar, es decir, mostrar un entendimiento rudimentario y básico de los conceptos. Aquí encontraríamos: presentación de conceptos con formato de clase magistral (apoyada con proyector de diapositivas), trabajo sobre imágenes, manipulación de simuladores, lectura de noticias relacionadas y comentarios de opiniones en grupo, actividades con plantilla para afianzar los conceptos expuestos.

\subsubsection{Enfoque role-play.}

Siguiendo con las ideas de Bloom y revisiones posteriores, estas actividades se concentraron en los ámbitos cognitivos de "aplicar, analizar, evaluar y crear" en los cuales el alumnado debe realizar tareas de pensamiento de orden superior (HOT, o High Order Thinking). En estos casos se siguen metodologías basadas en acciones del tipo: dramatizar, experimentar, examinar, practicar, aplicar, mostrar, dibujar, calcular, diferenciar, debatir, construir, explicar, inspeccionar, comparar, valorar, encuestar, decidir, comprobar, criticar, diseñar, confeccionar, elaborar, producir, inventar, idear, verificar, etc. Aquí encontraríamos: desarrollo de un proyecto personal ("La empresa en la que yo me veo trabajando", actividad que se llevó a cabo desde el primer hasta el último día de clase y donde el alumnado se implicó para completar las actividades del dossier así como en la escritura de una introducción y objetivos personales del curso así como una opinión y conclusión personal a la finalización del mismo), la realización de prácticas de laboratorio, debates, dinámicas de rol y dramatización de situaciones.

\subsection{Encuesta.}

Un año después de haber concluido el curso, se llevó a cabo una encuesta telemática dirigida al conjunto de estudiantes que había superado la formación. Se trató una población muestral de 12 personas. Para la encuesta se empleó la aplicación Formularios Google, dentro de la sitio Drive (Google ${ }^{\circledR}$ ). Se plantearon 5 preguntas referidas a conceptos trabajados con dinámicas clásicas y 5 referidas a las mismas áreas temáticas trabajadas con dinámicas de role-play. Cada una con tres posibles respuestas, solo una correcta. La encuesta se diseñó para que fuera totalmente anónima a fin de favorecer la participación de todos los estudiantes (algunos con posibles reticencias de privacidad). De hecho, ni siquiera el docente era capaz de conocer de quién era cada una de las contestaciones recibidas. 


\section{Resultados}

A continuación se muestran las preguntas que se formularon a los estudiantes en la plataforma digital, las posibles respuestas que se les ofrecieron y las respuestas obtenidas agrupadas en forma de gráfico de tarta. Para facilitar la comparación entre metodologías se han emparejado las preguntas por temática, donde la primera cuestión de cada grupo se refiere al concepto trabajado siguiendo la metodología de interés de este trabajo y la segunda aplicando alguno de los enfoques clásicos.

\subsection{Concepto de concentración de una disolución.}

En este primer bloque se presenta las cuestiones relacionadas con el concepto de concentración de una disolución que se trataron en el módulo del curso MF 1312 "Operaciones auxiliares y elementales en el laboratorio y en procesos en la industria química y afines". La metodología role-play fue aplicada en 3.1.1 como un juego espacial. Tomando el aula como recipiente: Los estudiantes de azul jugaban el papel de disolvente y los de camiseta de color correspondían a los distintos solutos.

\subsubsection{Pregunta formulada, opciones posibles y respuestas obtenidas con la metodología role-play.}

"Es posible que recuerdes la actividad que hicimos en el aula donde algunos vinisteis con camiseta azul, otros roja y alguno negra. ¿Cuál de las siguientes respuestas se adapta mejor al concepto de CONCENTRACIÓN de una mezcla formada por una sal disuelta en agua?"

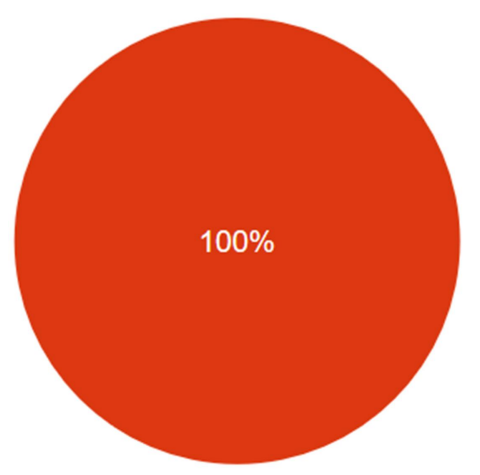

La concentración se refiere a cómo de centrados se encuentran los cristales de sal en el fondo del recipiente con agua.

La concentración se refiere a cuánta cantidad de sal se ha disuelto en el agua.

La concentración se refiere a cuánta agua se ha utilizado para disolver toda la sal.

\subsubsection{Pregunta formulada, opciones posibles y respuestas obtenidas con la metodología clásica.}

"Cuando estudiamos el concepto de CONCENTRACIÓN, aprendimos a diferenciar los distintos componentes. Si te dijesen que se ha disuelto una cantidad de sal en un volumen de agua, ¿qué afirmación sería la correcta?"

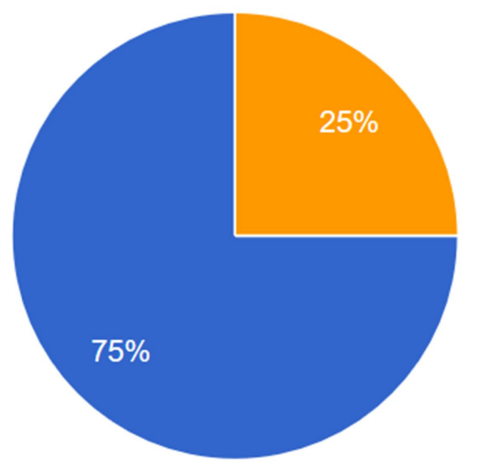

Se llama "disolvente" al agua y "soluto" a la sal.

Se llama "disolución" al agua y "soluto" a la sal.

Se llama "disolvente" al agua y "disoluto" a la sal.

(c)) EY-NC-No 2021, Universitat Politècnica de 


\subsection{Concepto de ley de prevención de riesgos laborales.}

Siguiendo con el temario del curso, en el módulo MF1311 "Operaciones de almacén de productos químicos y relacionados", se trató la cuestión de la Ley de prevención de riesgos laborales 31/1995 (8 de noviembre). Para profundizar en dicha ley y sus contenidos se llevó a cabo una actividad en grupo donde se intentó redactar uno de los apartados de la misma. La metodología role-play fue aplicada en 3.2.1 como un trabajo de búsqueda, reflexión y redacción en pequeño grupo o comité (cada miembro con un papel) seguido de un debate general con el resto de comités hasta llegar a un consenso.

\subsubsection{Pregunta formulada, opciones posibles y respuestas obtenidas con la metodología role-play.}

"Durante la dinámica donde trabamos la Ley de prevención, imaginasteis que formabais parte del comité técnico designado por el Ministerio de Empleo y Seguridad Social para redactar la actual Ley de Prevención de Riesgos Laborales. ¿Te viene a la memoria cómo definisteis el concepto de EQUIPO DE

PROTECCIÓN?"

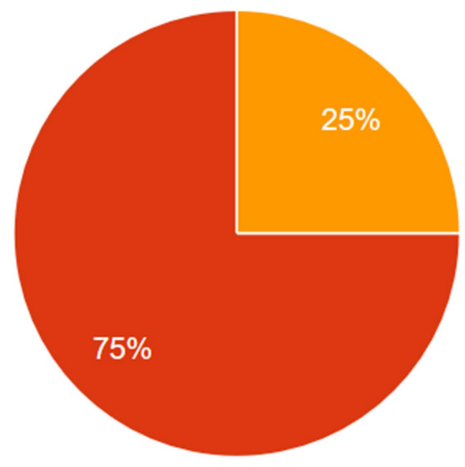

Cualquier equipo que proteja al trabajador/a de un riesgo inminente que pueda amenazar su seguridad en el trabajo.

Cualquier equipo que proteja al trabajador/a para que le proteja de uno o varios riesgos que pueda amenazar su seguridad o salud en el trabajo.

Cualquier equipo que proteja al trabajador/a de un riesgo que pueda amenazar su seguridad en el trabajo.

\subsubsection{Pregunta formulada, opciones posibles y respuestas obtenidas con la metodología clásica.}

"Los días que dedicamos a la descripción de los sistemas EPI de protección, hablamos de: tipos, modelos y usos. ¿Cuál te suena más cierta de estas tres afirmaciones?"

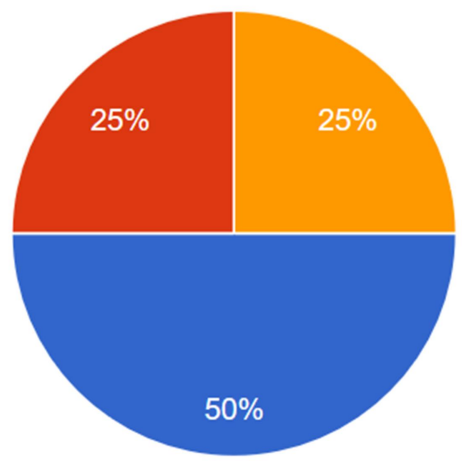

Es posible llevar gafas de vista durante una operación de riesgo siempre que se utilice la protección facial adecuada.

- Las máscaras contra polvo y olores que más protegen al usuario son las FPP1, las segundas que más le protegen son las FPP2 y las que menos las FPP3.

Un código de colores, visible en el filtro de una máscara, especifica la fecha de caducidad máxima de dicho filtro. 


\subsection{Concepto de plan de emergencia.}

En este tercer bloque, perteneciente al módulo MF1310 "Limpieza y desinfección en laboratorios e industrias químicas", se desarrollaron conceptos relacionados con los procesos físico-químicos que tienen lugar en los diferentes procedimientos de limpieza y desinfección de áreas sensibles de un laboratorio o industria. Entre las ideas trabajadas, se hizo hincapié en la normativa de seguridad y protocolos de actuación ante un accidente (por vertido, incendio, situación incontrolada, etc.). La metodología role-play fue aplicada en 3.3.1 como una dinámica de rol donde un grupo de estudiantes actuó como comité de empresa, otro como sindicato de trabajadores y otro como auditores externos todos intentando investigar un accidente sucedido en una planta de productos químicos.

\subsubsection{Pregunta formulada, opciones posibles y respuestas obtenidas con la metodología role-play.}

"En la dinámica sobre PLAN DE EMERGENCIA ante un accidente, unos actuasteis como comité de empresa y otros como auditores externos. ¿Recuerdas a qué se referían las instrucciones PAS?”

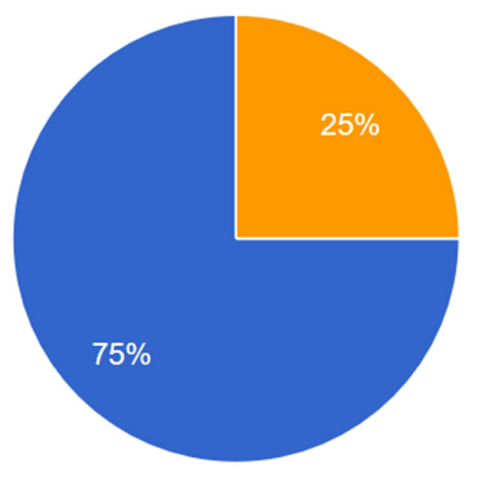

PAS son las siglas para Proteger-Alertar-Socorrer

PAS son las siglas para Proteger-AuxiliarSocorrer

PAS son las siglas para Prevenir-Acudir-Socorrer

\subsubsection{Pregunta formulada, opciones posibles y respuestas obtenidas con la metodología clásica.}

"Volviendo al PLAN DE EMERGENCIA, en él se recoge información sobre sobre operaciones con riesgo de vertido, ubicación de materiales absorbentes, persona responsable ante una emergencia, normas de actuación, etc. De entre las siguientes afirmaciones, ¿cuál piensas que falsa?”

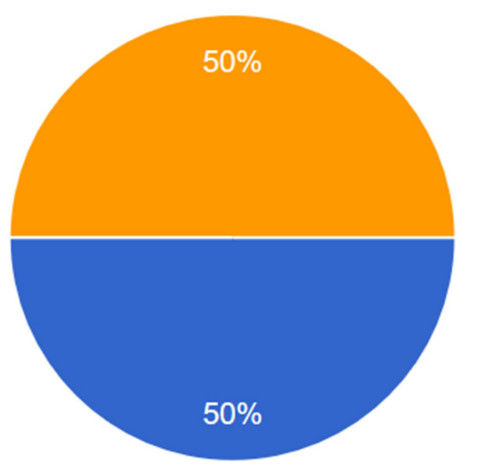

Las fichas de seguridad suelen tener entre $15 \mathrm{y}$ 20 apartados informativos según el país.

El Plan de emergencia debe incluir, al menos, la clasificación de los tipos de emergencia.

Las normas técnicas de prevención las publica el Instituto Nacional de Seguridad e Higiene en el Trabajo. 


\subsection{Concepto de transporte de mercancías.}

Durante una serie de sesiones se trabajaron conceptos e ideas relacionadas con la manipulación, gestión de documentación y normativa relacionada con el transporte de mercancías (siempre de naturaleza química), según requerido por el módulo MF1311 "Operaciones de almacén de productos químicos y relacionados". La metodología role-play fue aplicada en 3.4.1 mediante un trabajo de dramatización. Se organizaron grupos de tres estudiantes: uno representando al vendedor, otro al transportista y el último al cliente. Se les dieron directrices secretas por escrito para que cada uno expusiera frente a los otros dos (y frente a la clase) lo que creía que había sucedido en una serie de situaciones, siempre procurando defender sus intereses en la transacción.

\subsubsection{Pregunta formulada, opciones posibles y respuestas obtenidas con la metodología role-play.}

“A lo largo de la sesión dedicada a los DOCUMENTOS PARA EL TRANSPORTE DE MERCANCÍAS, realizamos un teatro en grupitos de tres. Unos erais la empresa de origen, otros el transportista y otros la empresa destinataria. ¿Cuál de las siguientes afirmaciones es FALSA?”

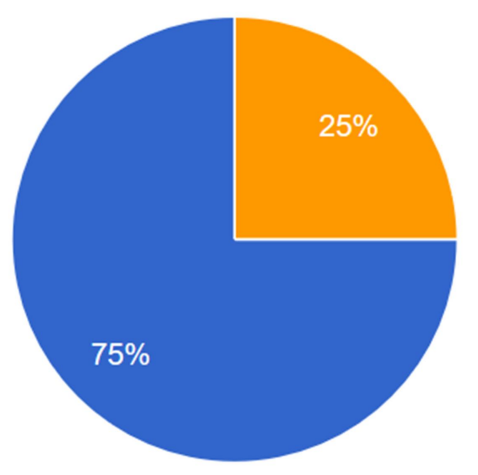

Un pedido solo puede ir asociado a un albarán y a una factura.

Los tres documentos fundamentales en un

proceso de compra-venta son: pedido, albarán y factura.

La cadena de compra-venta está formada por el remitente, el transportista y el destinatario.

\subsubsection{Pregunta formulada, opciones posibles y respuestas obtenidas con la metodología clásica.}

"Respecto al TRANSPORTE DE MERCANCIAS, dedicamos unas sesiones a hablar de los documentos y normativas que se aplican actualmente. Encuentra la opción FALSA:"

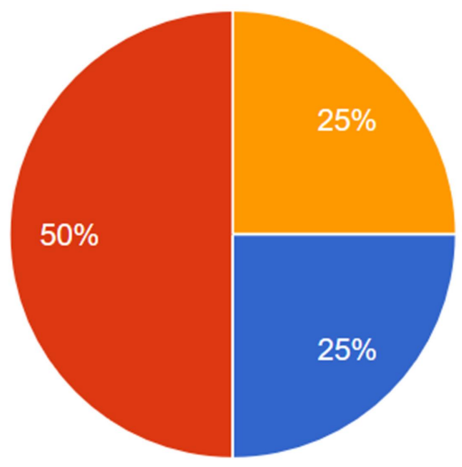

El reglamento REACH tiene entre sus objetivos mejorar la protección del medio ambiente.

La clasificación ADR diferencia las sustancias químicas según su riesgo con un código de números y letras.

La carta de porte puede sustituir al albarán cuando se contrata a un transportista externo. 


\section{E. Peris Chanzá}

\subsection{Concepto de normas de calidad.}

En este quinto y último bloque de preguntas, se trató el tema de las normas de calidad según las orientaciones del módulo MF1311. Aunque la visión sobre los sistemas de calidad solamente fuese superficial y descriptiva, se trató de dar una mirada global respecto a su importancia en el trabajo técnico dentro de la industria química, atendiendo a los cambios y mejoras que estas directrices de trabajo que han aportado. La metodología role-play fue aplicada en 3.5.1 a través de una sesión de debate partidista: se dividió la clase en cuatro grupos. Se les dio tiempo para buscar información y familiarizarse con una de las normativas. Después de un debate interno en busca de los puntos fuertes de su norma, se realizó una discusión general con el resto de grupos. El objetivo era o convencer al resto de grupos de la mayor importancia de su normativa o aceptar un consenso.

\subsubsection{Pregunta formulada, opciones posibles y respuestas obtenidas con la metodología role-play.}

"En el debate respecto a NORMAS DE CALIDAD, formasteis cuatro grupos: uno para la ISO 9001 (Gestión de calidad), otro para ISO 14001 (Gestión del medioambiente), otro para ISO 27001 (Gestión de riesgos y seguridad) y el último para ISO 18001 (Gestión de responsabilidad social). ¿Recuerdas a qué gran conclusión se llegó?"

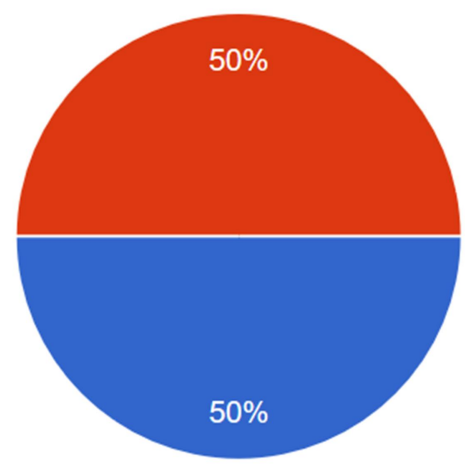

La ISO 9001 es la norma más importante de las cuatro porque es de la que surgen las demás.

Un Sistema Integrado de Calidad necesita de la combinación de, al menos, estas cuatro normas.

Un Sistema Integrado de Calidad está

compuesto por las ISO 9001 y 14001, y circunstancialmente por las ISO 27001 y 18001.

\subsubsection{Pregunta formulada, opciones posibles y respuestas obtenidas con la metodología clásica.}

"Para terminar este segundo bloque, ahí va una cuestión sobre NORMAS DE CALIDAD. Parecen todas ciertas pero una tiene un gazapo. ¿Cuál?”

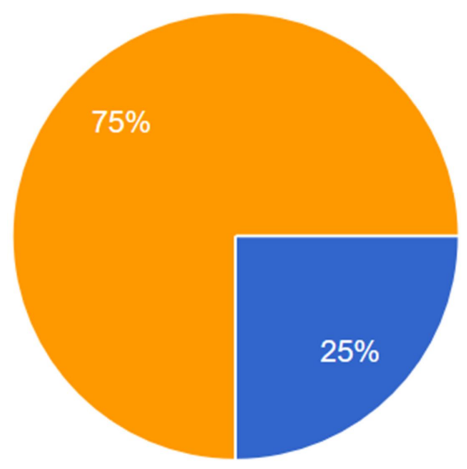

AENOR es el organismo estatal encargado de redactar las normas ISO.

En el nombre "UNE-ISO 9001", UNE son las siglas para Una Norma Española.

La norma ISO 9001 está orientada a Sistemas de gestión de la calidad.

(c)) BY-NC-No 2021, Universitat Politècnica de 


\subsection{Análisis de datos.}

\subsubsection{Grado de acierto.}

En primer lugar se muestra en la Tabla 1 la rúbrica para la corrección de las respuestas obtenidas a través de la encuesta:

\begin{tabular}{c|cc}
\multicolumn{3}{c}{ Tabla 1. Rúbrica con las respuestas correctas. } \\
\hline Pregunta & \multicolumn{2}{|c}{ Respuesta correcta } \\
\cline { 2 - 3 } $\mathbf{N}^{\mathbf{0}}$ & Role-play & Clásico \\
\hline 1 & Rojo & Azul \\
2 & Rojo & Amarillo \\
3 & Azul & Azul \\
4 & Azul & Amarillo \\
5 & Rojo & Azul \\
\hline
\end{tabular}

A partir de esta plantilla de resultados correctos, se analizaron las respuestas obtenidas considerando si fueron contestadas correcta o incorrectamente según cada una de las metodologías.

Tabla 2. Análisis del grado de acierto según la metodología.

\begin{tabular}{c|cc|cc}
\hline \multirow{2}{*}{$\begin{array}{c}\text { Pregunta } \\
\mathbf{N}^{\mathbf{0}}\end{array}$} & \multicolumn{2}{|c|}{$\mathbf{N}^{\mathbf{0}}$ de aciertos } & \multicolumn{2}{c}{ Aciertos (\%) } \\
\cline { 2 - 5 } & Role-play & Clásico & Role-play & Clásico \\
\hline 1 & 12 & 9 & 100 & 75 \\
2 & 9 & 3 & 75 & 25 \\
3 & 9 & 6 & 75 & 50 \\
4 & 9 & 6 & 75 & 50 \\
5 & 6 & 3 & 50 & 25 \\
\hline
\end{tabular}

Para observar mejor estos resultados se representaron como se muestra en la Figura 1. Lo principal que llamó nuestra atención, y motivo por el que se hizo este estudio, fue constatar que independientemente de la pregunta siempre se consiguió un mayor porcentaje de acierto cuando el concepto había sido trabajado mediante alguna dinámica role-play. Por el contrario, el aprendizaje en base a patrones más clásicos, resultó en mayor grado de error en las respuestas de los estudiantes. Aunque los resultados corresponden a una muestra de pocos datos, están orientados hacia el consenso generalizado que propone que el aprendizaje cooperativo, donde el estudiante debe participar activamente en el propio proceso de producción del conocimiento (frente a aquel aprendizaje menos participativo y centrado esencialmente en el despliegue de conceptos) produce una huella más duradera en el individuo, no solo desde el punto de vista de bienestar y gratitud por las propias dinámicas sino desde la fijación de los conceptos de una manera más natural y certera; lo que se evidencia en este estudio donde se comprueba como todos los conceptos llevados a cabo mediante role-play fueron retenidos mejor incluso después de un año desde el fin de las actividades. Este resultado tiene más importancia, si cabe, porque, como se comentó 


\section{E. Peris Chanzá}

anteriormente, gran parte de los estudiantes no tenían ninguna relación con el ámbito laboral ni académico del curso en cuestión.

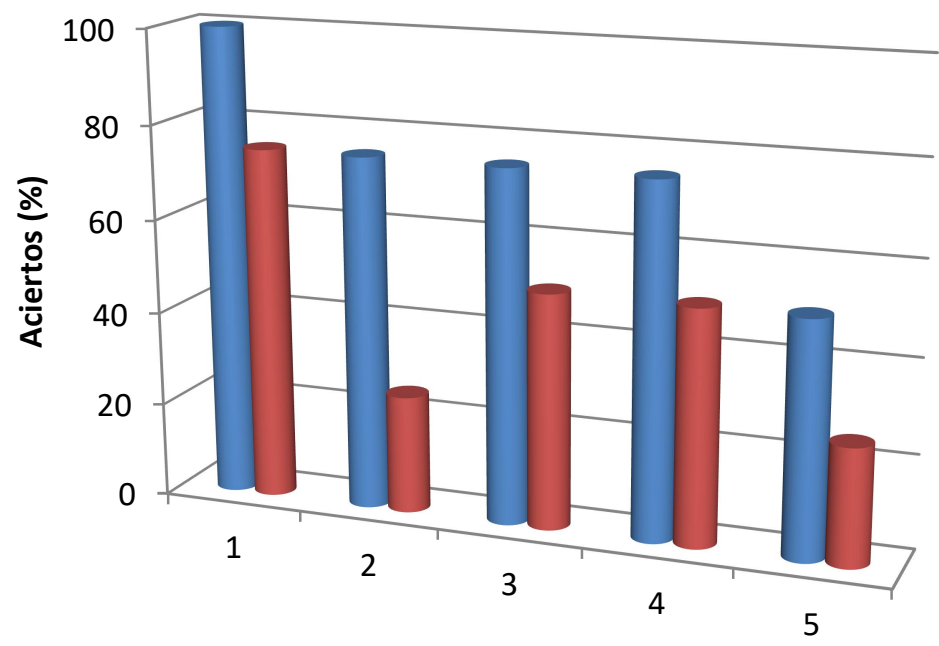

№ de pregunta

Fig. 1. Porcentaje de aciertos para cada pregunta según la metodología aplicada: role-play (azul) y clásica (rojo).

\subsubsection{Nivel de dispersión.}

Se pensó en la utilidad del valor del número de respuestas distintas obtenidas para cada pregunta como un descriptor del grado de duda y/o seguridad entre los alumnos a la hora de decidir entre una u otra repuesta, es decir, del nivel de afianzamiento de cada concepto. Los resultados se recopilaron según se ofrece en la Tabla 3 en base al número de respuestas distintas obtenidas para cada pregunta (pudiendo variar dentro del rango: 0 a 3) y para cada metodología.

Tabla 3. Análisis del grado de dispersión según la metodología.

\begin{tabular}{c|cc|cc}
\hline \multirow{2}{*}{$\begin{array}{c}\text { Pregunta } \\
\mathbf{N}^{\mathbf{0}}\end{array}$} & \multicolumn{2}{|c|}{$\mathbf{N}^{\mathbf{0}}$ de respuestas distintas } & \multicolumn{2}{|c}{ Dispersión (\%) } \\
\cline { 2 - 5 } & Role-play & Clásico & Role-play & Clásico \\
\hline 1 & 0 & 2 & 0 & 66 \\
2 & 2 & 3 & 66 & 100 \\
3 & 2 & 2 & 66 & 66 \\
4 & 2 & 3 & 66 & 100 \\
5 & 2 & 2 & 66 & 66 \\
\hline
\end{tabular}

Como sucediera con el estudio del nivel de acierto, también aquí se observó que existía una mayor homogeneidad en las respuestas cuando se planteaban desde la óptica de las sesiones llevadas a cabo aplicando las técnicas role-play con respecto a los enfoque clásicos. Esto es precisamente lo que muestran los resultados asociados a las preguntas 1,2 y 4, donde el nivel de dispersión aumenta con el enfoque clásico respecto al role-play. En el caso de las preguntas 3 y 5 , en ambas se obtuvieron dos respuestas 
distintas independientemente de la metodología puesta en marcha. Nuestra explicación para este comportamiento tiene varios enfoques. Por una parte, suponemos dentro del error del estudio el que alguna de las preguntas no consiga el resultado deseado; bien por que sea un concepto poco utilizado por los estudiantes (quizás ninguno ha tenido que volver a usarlo después de participar en las prácticas de final de curso), bien porque fuese nuevo para ellos en el momento de aprenderlo y no se reforzara lo suficiente durante las sesiones destinadas a ese módulo formativo o bien por la manera en la que fueron redactadas las preguntas de la encuesta. A este respecto, y aun cuando esta no fuese la fuente principal de la posible desviación en los resultados, vimos lógico (una vez ya lanzada la encuesta y recibidas las respuestas) que aquellas preguntas donde se manejan expresiones de negación suelen resultar más complejas de descifrar por los estudiantes. Así, por ejemplo, en la pregunta 3 "de entre las siguientes afirmaciones ¿cuál es falsa?” o en la pregunta 5 "parecen todas ciertas pero una tiene un gazapo, ¿cuál?" la propia redacción puede llevar a equívoco si la lectura no se realiza de manera atenta. Esto lo justificaríamos por el uso de las palabras "afirmaciones" y "falsa" (en la pregunta 3) o de "ciertas" y "gazapo" (en la pregunta 5). Por el contrario, en la pregunta 4, donde también se pedía que encontraran la opción incorrecta, la tipografía y el planteamiento de las cuestiones no dejaba lugar a dudas: en la pregunta 4 para la metodología role-play se podía leer "encuentra la opción FALSA" y para la metodología clásica “¿Cuál de estas afirmaciones es FALSA?” Por todo ello, si bien el nivel de dispersión en cuanto al número de respuestas distintas para cada metodología apunta en la línea de los resultados obtenidos según el porcentaje de acierto, se puede extraer una autocrítica al trabajo en la línea de redacción (fondo y forma/tipografía) de las cuestiones. Esto cobra más relevancia cuando la encuesta se pasa tiempo después de la finalización de las tareas del curso, por lo que es un punto a mejorar para futuras ediciones del mismo.

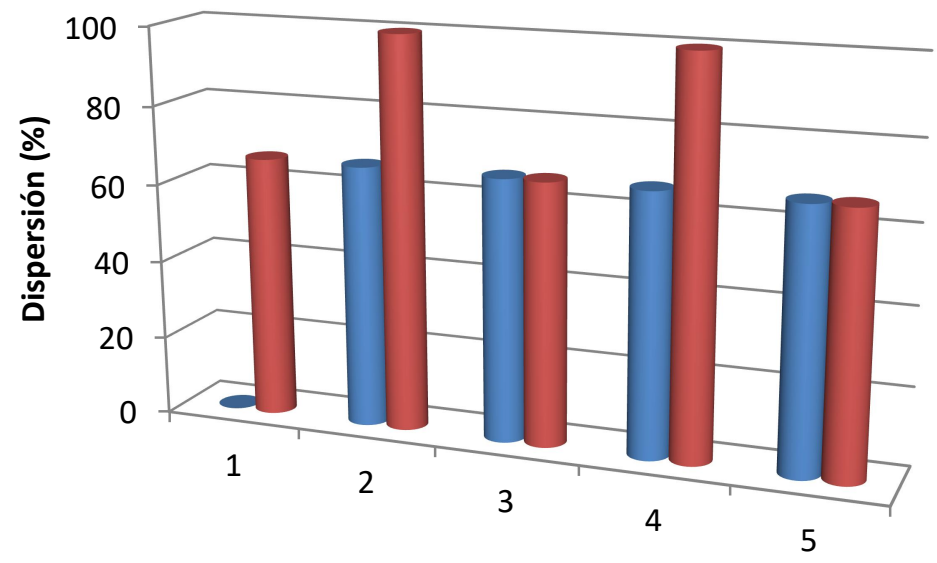

№ de pregunta

Fig. 2. Porcentaje de dispersión como porcentaje de variabilidad en el número distinto de respuestas obtenidas para cada pregunta y metodología aplicada: role-play (azul) y clásica (rojo). 


\section{E. Peris Chanzá}

\section{Conclusiones}

El análisis de los datos obtenidos a través de encuesta on-line realizada sobre el grupo de estudiantes un año después de la culminación de curso apunta en la dirección de que las metodologías role-play son más eficientes y fiables en cuanto a la permanencia de los conceptos trabajados, frente a los métodos tradicionales de enseñanza-aprendizaje. Así, el nivel de porcentaje de acierto para cada pregunta siempre aumentó con los métodos role-play. De manera similar, el nivel de dispersión en cuanto al número de respuestas distintas para cada pregunta generalmente fue menor con los métodos de interés. Ello permite apuntar a este conjunto de prácticas como adecuadas y recomendables para conseguir un aprendizaje duradero de conceptos, ideas y procedimientos que acompañen al individuo a lo largo de su vida. El aprendizaje vivencial deja huellas más profundas que cualquier otra forma de interacción tradicional con la información.

También se concluye haciendo una valoración crítica de la comunicación presentada que apunta a tres líneas: la necesidad futura de trabajar con mayor volumen de datos para obtener resultados más representativos y robustos, la utilidad disponer de un grupo control con el que comparar tendencias y la optimización en la manera de redactar las preguntas (fondo y forma) que, como se discutió en la sección anterior, puede jugar un papel condicionante en la respuesta.

\section{Referencias}

ANDERSON, L. W., KRATHWOHL, D. R. (2001). A taxonomy for learning, teaching and assessing : a revision of Bloom's taxonomy of educational objectives. New York: Longman.

BIGGS, J., TANG, C. (2011). Teaching for quality learning at university (4th ed.). Maidenhead, England: Open University Press (4th ed.).

BLOOM, B. S., ENGELHART, M. D., FURST, E. J., HILL, W. H.M KRATHWOHL, D. R. (1956). Taxonomy of educational objectives: the classification of educational goals. New York : David McKay Company (p. 201-7).

COFFIELD, F. (2008). Just suppose learning and teaching became the first priority. London: Learning and skills network. Available at: <http://tlp. excellencegateway.org.uk/ecpd/ecpd_modules/downloads/coffield_if_only.pdf>

Comunidad Valenciana. Decreto 51/2018, de 27 de abril, del Consell, por el que se modifica el Decreto 87/2015, por el que se establece el currículo y desarrolla la ordenación general de la educación secundaria obligatoria y del bachillerato en la Comunitat Valenciana.

España. Real Decreto 1105/2014, de 26 de diciembre, por el que se establece el currículo básico de la Educación secundaria obligatoria y del bachillerato (BOE 3 enero 2015) y decretos de currículos autonómicos.

Europa. Orden ECD/65/2015, de 21 de enero, por la que se describen las competencias, contenidos y criterios de evaluación de la Educación primaria, secundaria obligatoria y bachillerato.

GREEN, D., BLASZCZYNSKI, C. (2012). «Effective strategies and activities for developing soft skills» en Journal of Applied Research for Business Instruction. Ed. Delta Pi Epsilon (2012, 10(2)).

McSHARRY, G., JONES, S. (2000). «Role-play in science teaching and learning» en School Science Review (2000, $82,73-82)$.

RACE. P. (2010). Making learning happen: a guide for post-compulsory education (2 ${ }^{\text {nd }}$. ed.). London, England: SAGE Publications Ltd.

REEVES, T. C., HERRINGTON, J., OLIVER, R. (2002). «Authentic activities and online learning» en Annual Inernational Conference of the Higher Education Research and Development Society of Australasia (HERDSA). Perth, Autralia. Available at:

$<$ http://researchrepository.murdoch.edu.au/7034/1/authentic_activities_online_HERDSA_2002.pdf $>$ 
El role-play como ejemplo de aprendizaje duradero en población adulta

THE FLIPPED CLASSROOM (2015). La taxonomía de Bloom revisada y con una buena colección de verbos. $<$ https://www.theflippedclassroom.es/la-taxonomia-de-bloom-revisada-y-con-una-buena-coleccion-de-verbos/> [Consulta 22 marzo 2020]. 